\title{
Motivación en Ciencias del Deporte.
}

Autores: Pedro Moreno López-Navarrete, Fernando Durán Correa y Alejandro de la Lastra Ferrer-Egea.

Afiliación Alumnado asignatura "La educación en valores y práctica de actividad física en colectivos en situación de desigualdad." Grado de Ciencias del Deporte. Universidad de Huelva

Email: pedru1305@gmail.com / fernandodurancorrea@gmail.com / ale esp 10@hotmail.com 


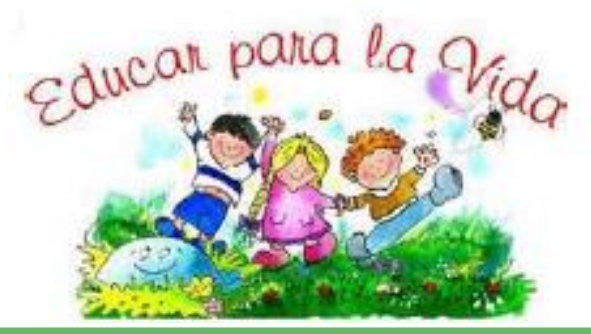

\section{Editorial \\ 2. Pregunta del dia}

3. Objetivos

4. Sesiones

5. Motivación.

6. Para saber más $\rightarrow$ libros

7. Atumento de la motivación y la adherencia en la enser̃anza deportiva.

8. Información adicional online

9. Test de psicologia deportiva

10. Reflexión

11. Teoria Autodeterminación

12. Como mejorar el clima motivacional a través del TARGET.

13. Articulos

14. Entrevista sobre motivación.

15. Conclusiones

16. Bibliografia y enlaces de interés.

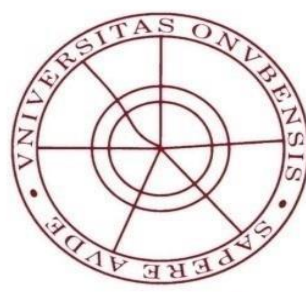

Eniversidad de Huelva 


\section{Editorial}

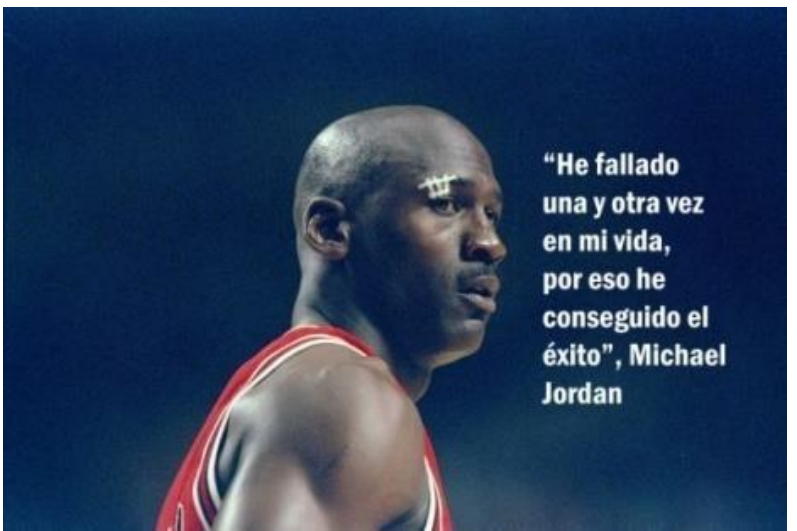

En este, nuestro boletín, realizado según la práctica número cuatro del curso, el principal hecho es la motivación, concebida como el énfasis que descubre una persona hacia la realización de una acción.

Otros autores definen la motivación como: los factores o determinantes internos que incitan a una acción. La motivación es un estado interno que activa, dirige y mantiene la conducta.

Antes de iniciar dicha práctica, Pedro nos expuso que una cosa es la orientación o motivación que trae el alumno y otra es, el clima que genera el docente.

.Siguiendo en la primera parte, antes de comenzar la práctica, para él debía de haber un conjunto de normas para que la clase funcionase. Entre ellas destacamos:

.Saber usar el material que tiene cierto riesgo.

.Respetar la división del terreno.

.Hacer caso a la señal del profesor.

Dicho esto, expondremos la práctica realizada, así como artículos, entrevistas, enlaces de vídeos, test... relacionados con este tema.

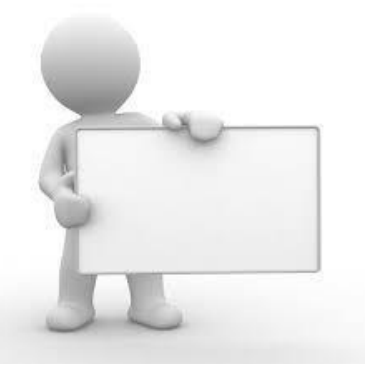

\section{Pregunta del día.}

¿Cómo motivar?--> Claves para la motivación.

\section{Objetivos de la práctica.}


onocer como docentes las claves fundamentales de motivación.

.Analizar cómo aumentar la motivación intrínseca a través de la satisfacción de las necesidades psicológicas básicas.

.Mejorar el clima tarea a través del TARGET.

.Conocer de forma externa la propia motivación (entrevista sobre la motivación).
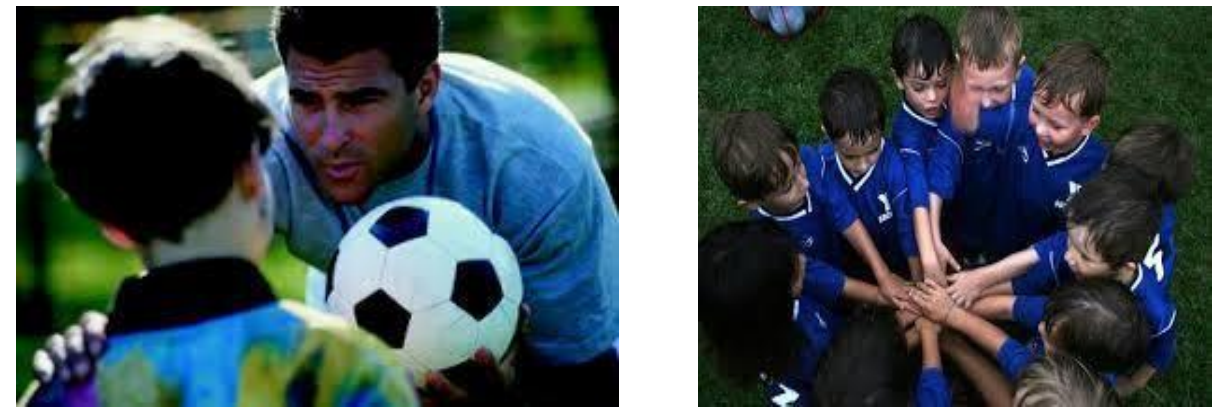

\section{Sesiones.}

ca la realizamos la clase entera, por lo que el profesor nos dividió (grupos A y B), en dos sesiones distintas, realizando el grupo A, la de Floorball y el grupo B la de raqueta.

En todo momento el profesor nos indicó que debíamos estar atentos, a la motivación y clima motivacional de cada una de las sesiones dadas de forma simultánea; para ello dividiendo la zona del pabellón en dos, cada una con su práctica.

Sesión de FloorBall (Grupo A).

Los objetivos que se buscaban en esta sesión, eran los de iniciación al floorball, los materiales utilizados fueron pelotas y conos. Decir que esta clase iba destinada a alumnos a partir de los 11 años.

En la parte inicial de ambas sesiones fue la de explicar aspectos globales de motivación y normas básicas en cualquier deporte. Tras esto en dicha sesión en particular, los alumnos realizaron conducciones libres cada uno con su stick y pelota. 
riante: Lo mismo, pero molestando al resto de compañeros y quitándoles la pelota.

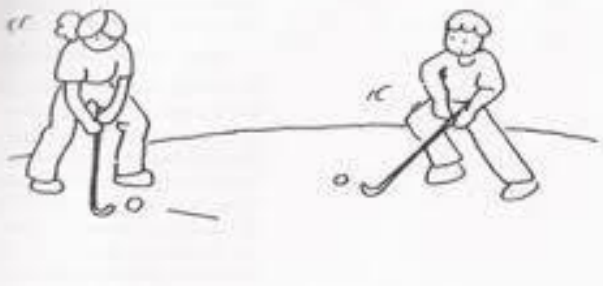

En la parte fundamental, realizaron varios ejercicios entre ellos:

-Por parejas, uno con pelota el otro se la intentaba quitar.

-Ratón y el gato. 3x1 Variantes: 2x2

-Preparación del partido grupal, y jugar 4x4 partido del floorball, utilizando los conos de porterías.

Tras finalizar la sesión como responsables de ver la motivación del grupo, podemos sacar las siguientes conclusiones:

-Al ser un deporte novedoso y no común los alumnos, han aprendido algunos aspectos técnico-tácticos referidos a este deporte y las normas básicas que conlleva cualquier deporte o actividad deportiva.

-De forma general, hemos visto que la

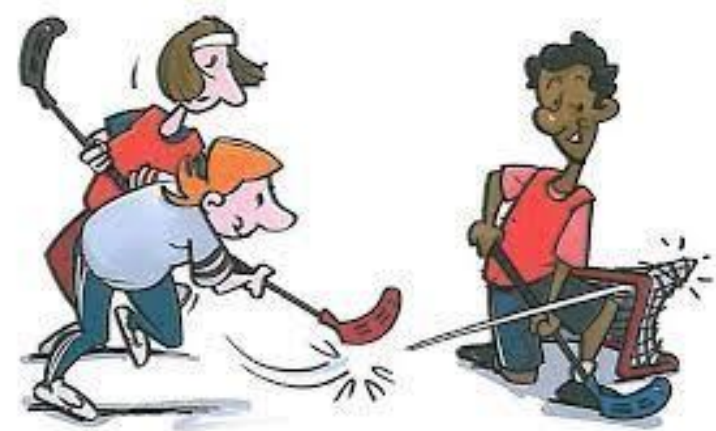
sesión les ha gustado y les ha parecido motivante, debido a como indicamos antes al ser un deporte menos común.

-Por último comentar que el comportamiento tanto de forma individual como grupal ha sido el adecuado.

Sesión Raqueta grupo B.

Simultáneamente, en la otra práctica:

En la parte inicial, aparte de las explicaciones objetadas anteriormente, realizamos algunos ejercicios como forma de calentamiento: 
e forma individual, cogíamos una raqueta y una pelota y debíamos darle toques sin que la pelota cayera al suelo.

-Variante: ahora nos pasábamos la pelota con un compañero (sin que esta cayera).

Ya en la parte principal, por parejas, delimitando un pequeño campo, jugar un pequeño partido (en mi caso no se podía hacer mates) para hacerlo más sencillo.

Rondos en la que uno se la quedaba en medio y tres tenían que pasarse la bola sin que el del medio se la quitara.

Partido 4vs4 delimitado por una red (podía dar un bote, mínimo tres toques, poniendo en muchos casos las normas nosotros).

En la sesión de raqueta, en general la motivación no fue excesiva (debido a la menor complejidad de los ejercicios, reflejando algo más en el partido final o en los rondos).

- Sáenz-López, P. (2015). Apuntes inéditos de la asignatura "la educación en valores y la práctica de actividad física en colectivos en situación de desigualdad".

Ciencias de la Actividad Física y del Deporte. Universidad de Huelva.

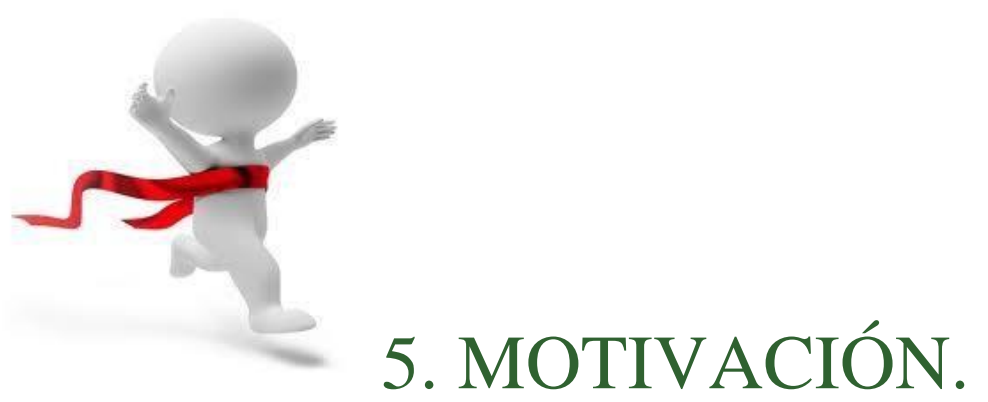

La motivación se puede definir como el conjunto de variables sociales, ambientales e de esa actividad, la persistencia en la tarea y el rendimiento.(Núñez Alonso y Martín-Albo Lucas).

Para Atkinson y Feather (1966), en la propia motivación, venia determinado por el motivo de la tendencia al logro "esperanza de éxito", como un motivo negativo, denominado temor al fracaso.

Por tanto, dentro de la actividad física ¿Qué técnicas debemos seguir para incrementar la motivación? Según Weinberg (1994) serían:

1. Asegurarse de que todos, tienen oportunidad de experimentar éxito en ocasiones. Para ello los entrenadores deben diseñar actividades específicas para sus deportistas. 
crementar la percepción de control, haciéndoles partícipes del proceso de decisión y dándoles responsabilidades.

3. Utilizando refuerzo positivo y aprobación siempre que sea posible.

4. Establecer objetivos alcanzables pero que representen un reto.

5. Asegurar variedad en el programa de entrenamiento. Facilitar la comunicación, fomentar el sentido de orgullo en pertenecer al grupo, establecer normas comunes de conducta, valorar las contribuciones individuales.

Todos estos puntos sobre la motivación, es conveniente objetar, que son definidos según la motivación en la actividad física y deportiva.

\section{Para saber más...}

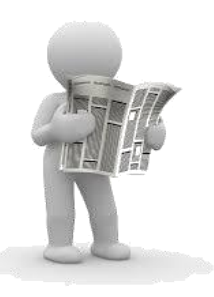

\section{Libros.}

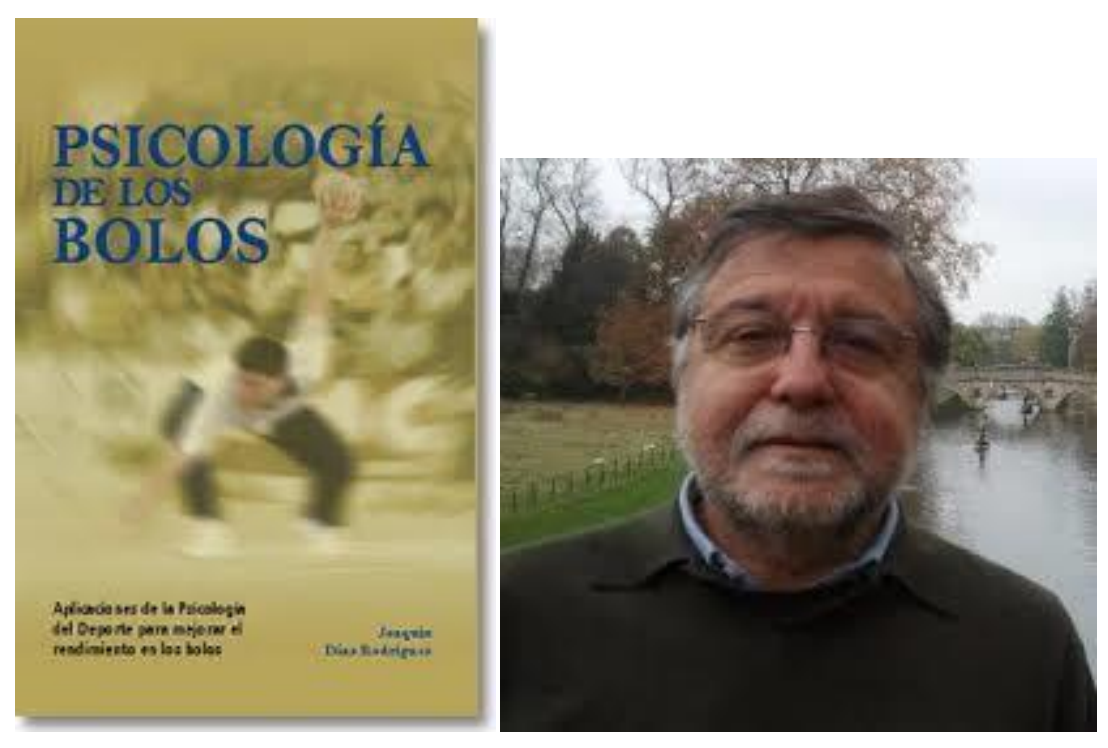

Joaquín Díaz (2003).

Como cualquier otro deporte, el juego de los bolos a alto nivel requiere jugadores con una actitud mental positiva, que no piensen en el error y que jueguen con confianza, determinación y seguridad. Balague,(2002). 
aquín Díaz Rodríguez, en su libro psicología de los bolos, expone un caso de un jugador de bolos, de la modalidad bolo-palma de primera liga nacional; el cual, ha sido tres veces campeón de España individual, pero como casi todos los jugadores de bolos no tienen entrenador, ni asesores, por lo que sus estados anímicos y deportivos debe generarlo el mismo.

Sus malos resultados esta temporada, le hacen ir a un psicólogo, preguntándose, porque lo pasa mal y sufre en la pista, en sí su forma de tiro técnicamente era la misma de siempre, pero lo que había que recuperar era la confianza y automotivación.

Con ello podemos concluir que en deportes individuales, los aspectos mentales son fundamentales para conseguir los objetivos previstos más allá de la propia técnica.

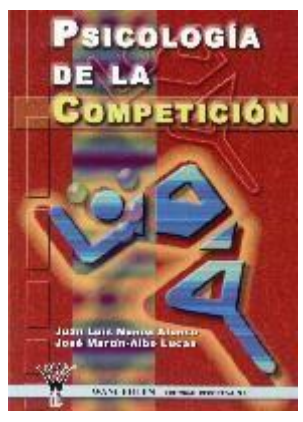

José Martín Albo y Juan Luis Núñez Alonso (2004).

La alta competición requiere un alto grado de especialización en todos los ámbitos deportivos, lo que se ha traducido en la aparición de numerosos expertos: médicos, nutricionistas, preparadores físicos..

Este libro escrito por Juan Luis Núñez Alonso y José Martín-Albo Lucas presentan la importancia del entrenamiento psicológico en el deporte competitivo, además recogen un conjunto de técnicas que más se utilizan en el entrenamiento de habilidades psicológicas deportivas, entre ellas destacamos: La motivación, el control de la ansiedad, el manejo de la atención y la concentración.

\section{Aumento de la motivación y la adherencia en la enseñanza deportiva.}

Esta tesis doctoral realizada por Bartolomé Jesús Almagro Torres (Becario FPU del Ministerio de Ciencia e Innovación) y dirigida por Pedro Sáenz-López Buñuel y Juan 
tonio Moreno Murcia, en ella, se nos expone como la propia motivación influye en la realización de AF y deporte.

Para Moreno, Cervelló y González-Cutre, (2006), la motivación es un elemento clave en el compromiso y la adherencia a la práctica físico-deportiva.

Conocer también que la adolescencia es un período clave para la adherencia de los jóvenes a la hora de seguir practicando actividad física o abandonarla por completo, teniendo que considerarse el propio entrenamiento como un tiempo de disfrute donde el chico se encuentre motivado, si no es así es difícil que este llegue a tomar el ejercicio como rutina diaria. Cervelló et al, (2007),

Finalmente en la misma se presentan algunas propuestas prácticas para aumentar la motivación en la práctica deportiva; para ello se buscan formas a la hora de diseñar o seleccionar las tareas motrices.

Según Sáenz-López y Giménez, (2000) es fundamental algunos de estos aspectos, de los cuales destacamos los siguientes:

-Duración y participación suficiente para que el alumno tenga un margen de asimilación personal.

-Búsqueda de situaciones reales.

-Estas actividades deben llevar al éxito de una forma u otra.

-Resolver los problemas motrices.

Otros aspectos fundamentales son el reconocimiento al esfuerzo, trabajo y comportamiento que el docente debe transmitir a los jugadores Martens (1989).

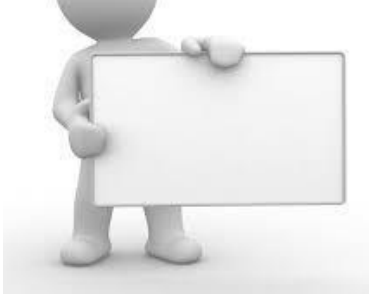

\section{Información adicional online}

\section{Entrevistas.}

-Entrevistas inicial a deportistas por Amparo Pozo Calvo: 
tp://www.saludmental.info/Secciones/deporte/2006/entrevista_inicial_deportistas_di c06.htm

-Entrevista a Leyre Santos, deportista internacional de saltos de trampolín:

http://www.psicologiadeportiva.net/revista/articulo/52.html-Videos:

https://www.youtube.com/watch?v=WVmdC6cJ-1c

-Persigue tu objetivo:

https://www.youtube.com/watch?v=3Y1Z4yx97kM

-Pep Guardiola y Fernando Trueba (director de cine) el Liderazgo y el Futuro:

https://www.youtube.com/watch?v=_LoDoTsCAS8-

Motivación en el deporte.

https://www.youtube.com/watch?v=UBT_odMLea4-Teoría

de autodeterminación:

https://www.youtube.com/watch?v=LB_pn8fkO3I

9. Test de

\section{deportiva:}

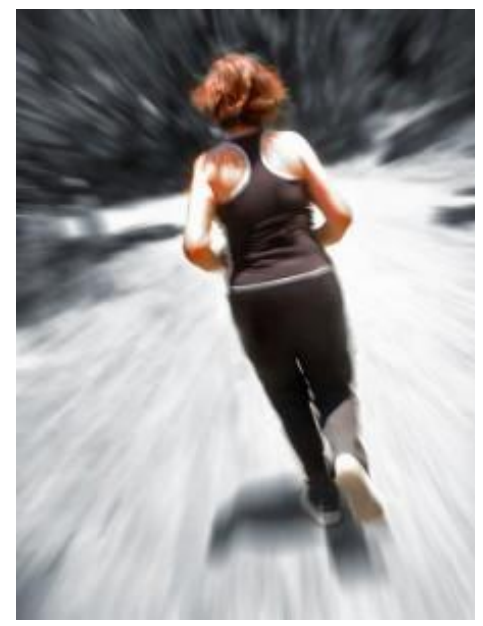

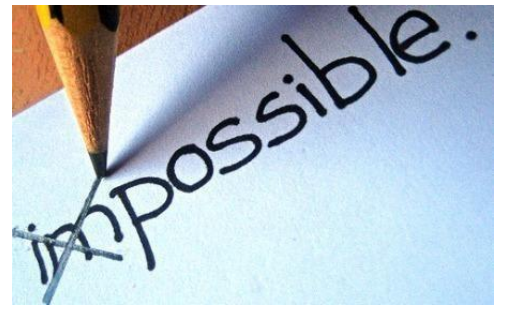

psicología

\section{Metas motivacionales.}

Cada deportista tiene un funcionamiento psicológico diferente y no puede ser comparado con los demás. No obstante conviene tener en cuenta algunas variables como la motivación, el estrés, la autoconfianza y el nivel de activación.

En este test que vamos a presentar a continuación, se exponen una serie de preguntas a la que debemos contestar del 1 al 7, siendo el 1 totalmente desacuerdo al 7 totalmente de acuerdo. 
Psicología deportiva. Conroy, D. E., Elliot, A. J., y Hofer, S. M. (2003).

$\begin{array}{lll}\text { To } & \text { Ba } & \\ \text { tall } & \text { sta } & \text { Al } \\ \text { me } & \text { nte } & \text { go } \\ \text { nte } & \text { en } & \text { en } \\ \text { en } & \text { de } & \text { de } \\ \text { de } & \text { sac } & \text { sa } \\ \text { sac } & \text { ue } & \text { ue } \\ \text { ue } & \text { rd } & \text { rd } \\ & \text { o } & \text { o }\end{array}$

Durante la práctica de actividades físicodeportivas... (2x2)

$$
\begin{aligned}
& \mathrm{Ne} \\
& \text { utr } \\
& \mathrm{O}
\end{aligned}
$$

Es importante para mí hacerlo mejor que los demás

\author{
(1)
}

(2)

Es importante para mí hacerlo tan bien como pueda

Simplemente quiero evitar hacerlo peor que los demás

A veces tengo miedo de no poder hacerlo tan bien como me gustaría

Es importante para mí hacerlo bien comparado con los demás

Quiero hacerlo tan bien como me sea posible

Mi meta es evitar hacerlo peor que todos los demás

A menudo me preocupa no poder hacerlo tan bien como puedo

Mi meta es hacerlo mejor que la mayoría de los otros/as practicantes

Es importante para mí dominar todos los aspectos de mi actuación

Es importante para mí evitar ser uno/a de los/as peores practicantes del grupo Me preocupa no poder hacerlo tan bien como posiblemente podría
(1) 2

(1)

2030

(6

7 $\begin{array}{lllllll}1 & 2 & 3 & 4 & 5 & 6 & 0\end{array}$

$\begin{array}{lllllll}1 & 2 & 3 & 4 & 5 & 6 & 0\end{array}$

$\begin{array}{llllll}1 & 2 & 3 & 4 & 5 & 6\end{array}$

$\begin{array}{lllllll}1 & 2 & 3 & 4 & 5 & 6 & 0\end{array}$

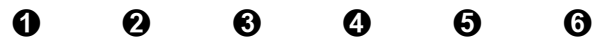

$\begin{array}{lllllll}1 & 2 & 3 & 4 & 5 & 6 & 0\end{array}$

$\begin{array}{lllllll}1 & 2 & 3 & 4 & 5 & 6 & 0\end{array}$

$\begin{array}{lllllll}1 & 2 & 3 & 4 & 5 & 6 & 0\end{array}$ 


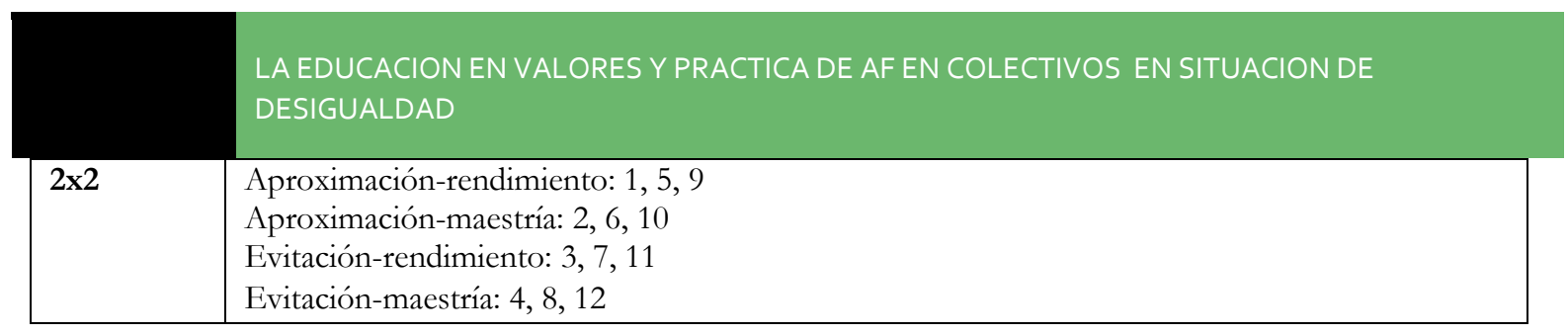

\section{Reflexión.}

Tras finalizar ambas sesiones, nuestro compañero Daniel Mihura(bajo su punto de vista), nos aportó que (para él) la sesión creía que había sido más motivante y que ejercicios o que metodología debemos de llevar a cabo para fomentar la motivación en el alumnado $\rightarrow$ Conforme avanza la sesión, la motivación va aumentando; el hecho de cooperar y competir (trabajar con gente), es uno de los aspectos fundamentales, más que trabajar solos.

Álvaro $\rightarrow$ nos dijo que para él, la sesión de foorball podía haber sido más motivante que la de raqueta (la que él hizo), debido a ser un deporte menos usual.

Pedro comento que en ocasiones, lo inusual o lo novedoso o incluso llevado a otro extremo lo peligroso hace que algunas personas les motive aún más; en cambio a otras justamente lo contrario (por ello decir que cada persona tiene una forma diferente de pensar, y de una forma u otra piensa que es normal como el actúa... (Normopatía $\rightarrow$ dada en la clase del miércoles, creyendo cada persona que es normal en sus actitudes o formas de actuar y pensar).

Pedro nos habló, sobre la teoría de autodeterminación que luego veremos más detenidamente.

Para finalizar esta reflexión, para nosotros una frase que nos ha gustado especialmente ha sido:

"El mejor placer en la vida es hacer lo que la gente te dice que no puedes hacer" Walter Bight.

\section{Teoría de la autodeterminación.}


La teoría de la autodeterminación, Deci y Ryan (2012), la definen como una teoría empírica de la motivación humana y la personalidad en los contextos sociales, que distingue entre motivación autónoma y controlada.

Esta teoría ha sido utilizada para explicar la motivación humana en diferentes contextos: en el trabajo, en el ámbito educativo, en el cuidado de la salud, en psicoterapia, etc. En este sentido, en los últimos años, la TAD ha sido ampliamente aplicada al ámbito de la actividad física y el deporte, ya que resulta de gran utilidad para analizar aspectos relacionados con la adherencia a la práctica deportiva.

Son 5 mini-teorías, de las cuales nos centraremos en solo una:

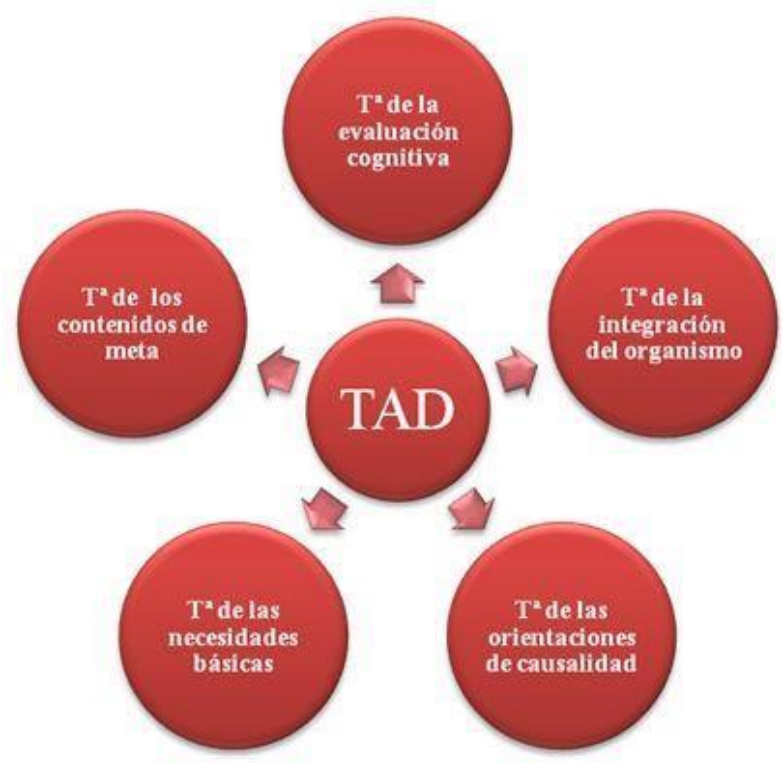

Teoría de las necesidades básicas. Esta mini-teoría aclara conceptos fundamentales para la teoría de la autodeterminación, como son las de las necesidades psicológicas básicas y su relación con la salud psicológica o el bienestar. Deci y Ryan, (1985, 2000); Ryan y Deci, (2000).

Esta teoría asume que existen tres necesidades básicas, innatas y universales, que son: la necesidad de competencia, la necesidad de autonomía y la necesidad de relación con los demás. Deci y Ryan, (1985, 2000).

Deci y Ryan (1991) definieron estas tres necesidades, del siguiente modo:

- Competencia. La necesidad de competencia se basa en tratar de controlar el resultado y experimentar eficacia.

- Autonomía. En lo que se refiere a la necesidad de autonomía, ésta comprende los esfuerzos de las personas por ser el agente, por sentirse el origen de sus acciones, y tener voz o fuerza para determinar su propio comportamiento.

- Relación con los demás. La necesidad de relación con los demás hace referencia al esfuerzo por relacionarse y preocuparse por otros, así como sentir que los 
demás tienen una relación auténtica contigo, y experimentar satisfacción con el mundo social.

Las investigaciones indican que cada una de ellas juegan un papel importante para el desarrollo y la experiencia óptima, así como para el bienestar en la vida diaria Ryan y Deci, (2000), de manera que ninguna puede ser frustrada sin consecuencias negativas, por lo que resulta necesaria la satisfacción de las tres necesidades. De hecho, en la teoría de la autodeterminación, las necesidades básicas constituyen los mediadores psicológicos que influirán en los tres principales tipos de motivación, así como en otras consecuencias (bienestar psicológico, autoestima, vitalidad, funcionamiento óptimo, etc.).

Cuanto más puedan mejorar estas 3 competencias los alumnos, mayor será su motivación acerca de la actividad.

Hacemos esencialmente las cosas en que nos sentimos componentes, esto es el ABC de la motivación $\rightarrow$ Pedro Sáenz-López Buñuel. (2014).

\section{Como mejorar el clima de la tarea a través del TARGET.}

Ames (1992), agrupó las dimensiones teóricas de una meta de maestría en seis escenarios de aprendizaje, identificados con el acrónimo inglés TARGET. Desglosando dichas siglas obtenemos las distintas palabras:

$\mathrm{T} \rightarrow$ Tarea.

$A \rightarrow$ Autoridad.

$\mathrm{R} \rightarrow$ Reconocimiento.

$\mathrm{G} \rightarrow$ Grupo.

$\mathrm{E} \rightarrow$ EvaluaciónT $\rightarrow$ Tiemp

o

Ames (1992).

Dicha palabra, podemos traducirla como meta u objetivo, y en base a esto recordamos que nuestro objetivo debería ser desarrollar el clima tarea. 
Para ello Ames (1992), expone las estrategias de motivación que deben ser empleadas para manipular las estructuras de metas ambientales y conseguir una implicación a la tarea de sujetos.

\begin{tabular}{|c|c|}
\hline DESCRIPCIÓN DE LAS ÁREAS & ESTRATEGIAS \\
\hline $\begin{array}{l}\text { Tarea } \\
\text { Diseño de las tareas y activida- } \\
\text { des }\end{array}$ & $\begin{array}{l}\text { Diseñar actividades basadas en la variedad, el reto } \\
\text { personal y la implicación activa } \\
\text { Ayudar a los sujetos a ser realistas y plantear objeti- } \\
\text { vos a corto plazo }\end{array}$ \\
\hline $\begin{array}{l}\text { Autoridad } \\
\text { Participación del sujeto en el } \\
\text { proceso instruccional }\end{array}$ & $\begin{array}{l}\text { Implicar a los sujetos en las decisiones y en los pape- } \\
\text { les de liderazgo } \\
\text { Ayudar a los sujetos a desarrollar técnicas de auto- } \\
\text { control y auto-dirección }\end{array}$ \\
\hline $\begin{array}{l}\text { Reconocimiento } \\
\text { Razones para el reconocimiento; } \\
\text { distribución de las recompensas; } \\
\text { oportunidades para las recom- } \\
\text { pensas }\end{array}$ & $\begin{array}{l}\text { Reconocimiento del progreso individual y de la mejora } \\
\text { Asegurar las mismas oportunidades para la obten- } \\
\text { ción de recompensas } \\
\text { Centrarse en el auto-valor de cada individuo }\end{array}$ \\
\hline $\begin{array}{l}\text { Agrupación } \\
\text { Forma y frecuencia en que los } \\
\text { sujetos interactúan juntos }\end{array}$ & $\begin{array}{l}\text { Agrupar a los sujetos de forma flexible y heterogé- } \\
\text { nea } \\
\text { Posibilitar múltiples formas de agrupamiento de los } \\
\text { individuos }\end{array}$ \\
\hline $\begin{array}{l}\text { Evaluación } \\
\text { Establecimiento de los estánda- } \\
\text { res de rendimiento. Guia del } \\
\text { rendimiento; feedback evaluati- } \\
\text { vo }\end{array}$ & $\begin{array}{l}\text { Utilizar criterios relativos al progreso personal y al } \\
\text { dominio de la tarea } \\
\text { Implicar al sujeto en la auto-evaluación } \\
\text { Utilizar evaluación privada y significativa }\end{array}$ \\
\hline $\begin{array}{l}\text { Tiempo } \\
\text { Flexibilidad de la programación. } \\
\text { Pasos del aprendizaje; dirección } \\
\text { del trabajo }\end{array}$ & $\begin{array}{l}\text { Posibilitar oportunidades y tiempo para el progreso } \\
\text { Ayudar a los sujetos a establecer el trabajo y la pro- } \\
\text { gramación de la práctica }\end{array}$ \\
\hline
\end{tabular}

Tabla de descripción de las tareas. Ames (1992).

De una forma resumida el deporte como entrenamiento y la propia competición, debe ir orientado hacia la tarea ya que esto ayudará a que se desarrollen formas de motivación más autodeterminadas así, como otras consecuencias positivas: competencia, disfrute, ...y con ello aumentará la probabilidad de que se comprometa uno mismo con la práctica de actividad físico-deportiva.

Por último recordar que el tiempo de enseñanza es fundamental para que se adquieran dichos conocimientos. 


\section{Artículos.}

A continuación, vamos a nombrar 3 artículos que resumimos sobre la motivación y un pequeño comentario englobando los tres:

ARTÍCULO 1 (Juan Antonio Moreno Murcia), Percepción de la utilidad e importancia de la educación física según la motivación generada por el docente.

ARTÍCULO 2: (Bartolomé Almagro y Pedro Sáenz-López) Clima motivacional percibido, necesidades psicológicas y motivación intrínseca como predictores del compromiso deportivo en adolescentes.

ARTíCUlO 3: (Juan Antonio Moreno Murcia, Eduardo Cervelló Gimeno y David González-Cutre Coll), Motivación autodeterminada y flujo disposicional en el deporte.

Comentario: En estos artículos se nos habla de la relación existente entre el alumno y el profesor en la percepción de utilidad e importancia de la Educación Física. Como ya hemos visto, la relación que existe entre los alumnos y los profesores además de ser abundante, es muy directa. Es decir, si los alumnos no se ven motivados por la metodología que el profesor utiliza, estos, no le darán mucha importancia a la consecución de los beneficios que supone la Educación Física a nivel físicopsicológicosocial. En cambio, si el profesor utiliza una metodología con la que los niños/as se vean identificados, estos, se implicarán al máximo para la correcta ejecución de los ejercicios y para la posterior consecución de los beneficios.

Por tanto, cada vez es mayor el peso que adquiere el profesorado sobre los alumnos en todos los ámbitos de la Educación, ya que, si tienes la capacidad de educar y divertir esto será más fácil que educar por "fuerza" o con metodologías que para nada son motivadoras para el alumnado.

Por tanto concluimos que la motivación y educación están claramente relacionadas.

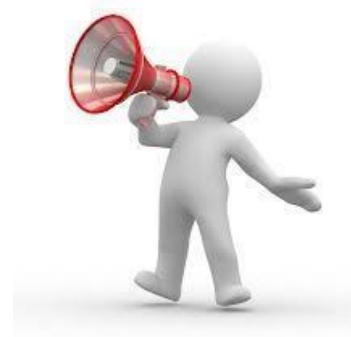

\section{Entrevista sobre motivación.}

Con este último apartado del Boletín, concluiremos con una pequeña entrevista realizada a Cristina Conde García (profesora nuestra de Enseñanza y Promoción de los 
Deportes de Raqueta) sobre la motivación y la intervención que ella realizo en su tesis sobre este tema.

La entrevista fue realizada por Fernando Durán Correa, Alejandro De La Lastra FerrerEgea y Pedro Moreno-López-Navarrete.

1. ¿Qué concepto tienes por motivación? ¿Y dentro del deporte?

Cristina: Para muchos autores y para mí en este caso, la motivación es el motor que hace que las personas inicien, modifiquen o abandones unas determinadas conductas.

Dentro del deporte, lo llevamos a que una persona inicie, mantenga o abandone la práctica deportiva.

2. ¿Cómo crees que puede influir estar motivado para realizar actividad física y deporte?

Cristina:Hay distintos tipos de motivación, desde la más intrínseca (realizo actividad física por disfrute personal), hasta la más extrínseca (realizo actividad física por un fin, este puede ser dinero..)

Decimos entonces que cuanto más intrínseca sea esta motivación, de forma más positiva realizaremos actividad física y por lo tanto, seguiremos realizándola a largo plazo.

3. ¿Qué aspectos destacarías en tu tesis?

Cistina:Mi tesis: Efectos de la intervención en el clima. Tarea sobre la motivación en jóvenes deportistas, se llevó a cabo una intervención. En ella diseñamos un programa llamado (PIFE), para entrenadores de baloncesto con el fin de realizar una estrategia para mejorar la motivación de los jugadores. El programa fue un éxito debido al cambio que se produjo tras su uso.

4. ¿Cómo docente utilizas la motivación en tus alumnos? ¿De qué forma?

Cristina:Para mí es fundamental, la utilización de las tres necesidades psicológicas básicas:

-Autonomía.

-Relaciones entre ellos.

-Competencia.

Las tres las utilizo como docente, hacia mis alumnos.

5. ¿De forma personal, alguna experiencia personal, referente a la motivación en la Educación Física?

Cristina: Una experiencia que me llevó a ser lo que soy hoy, docente de Educación Física, es debido a un profesor de E.F que tuve en primaria que utilizaba ya, estas tres 
necesidades psicológicas básicas (las nombradas anteriormente), y me motivó de tal forma que decidí dedicarme a este campo de la educación.

\section{Conclusiones.}

Tras todo lo visto, cabe mencionar la gran importación de la motivación en el ámbito deportivo, siendo esta un pilar fundamental para el inicio y mantenimiento a largo plazo de la actividad física.

Una vez leído artículos, buscado páginas webs sobre la motivación; no solo en el propio deporte sino en la vida, nos hemos dado cuenta como algo que ansias tener y luchas por ello lo consigues.

Finalmente en nuestra labor como docentes, teniendo un claro ejemplo, con la entrevista a nuestra profesora de Deportes de Raqueta Cristina, las 3 necesidades psicológicas básicas: Autonomía, competencia y relación con los demás usadas de una forma adecuada forman no solo a deportistas de alto nivel, sino a personas con principios para afrontar su día a día de la mejor forma posible.

Durán Correa, F.; De La Lastra Ferrer-Egea, A.; Moreno López-Navarrete, P.B. (2015). 


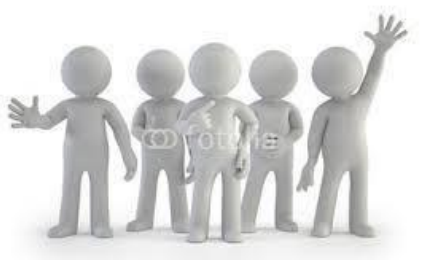

\section{Bibliografía y enlaces de interés.}

* Libros:

-Almagro Torres, Bartolomé Jesús; Aumento del clima motivacional hacia la tarea, (Becario FPU del ministerio de Ciencia e Innovación. Universidad de Huelva).

- Díaz Rodríguez, Joaquín, libros publicados sobre Psicología deportiva. http://www.psicologiadeporte.org/libros.html

* Artículos:

-Almagro, Bartolomé; Sáenz-López, Pedro; González-Cutre, David; Moreno-Murcia, Juan Antonio. Clima motivacional percibido, necesidades psicológicas y motivación intrínseca como predictores del compromiso deportivo en adolescentes: http://www.cafyd.com/REVISTA/02501.pdf

-Guía Fitness, http://guiafitness.com/frasesmotivadorashttp://www.papelesdelpsicologo.es/vernumero.asp?id=451

-Jornadas de EF en la Escuela: Factores motivacionales relacionados con la adherencia a la práctica en diferentes contextos físico-deportivos. Servicio de publicaciones de la Universidad de Huelva.

-Moreno-Murcia, Juan Antonio. Percepción de la utilidad e importancia de la educación física según la motivación generada por el docente:

http://www.revistaeducacion.mec.es/doi/362_165.pdf

-Moreno-Murcia, Juan Antonio; Cervelló Gimeno, Eduardo; González-Cutre Coll, David. Motivación autodeterminada y flujo disposicional en el deporte.

http://revistas.um.es/analesps/article/view/23291/22571

-Sáenz-López, P.; Conde, C.; Almagro, B.J. (2009). Aumento de la motivación y la adherencia en la enseñanza deportiva. En Sáenz-López, P.; Castillo, E.; Almagro, B.; Conde, C. y Gil, P. (Eds.). XVII 
* Web:

-Núñez

Alonso,

Juan

$\mathrm{y}$

Martín-Albo

Lucas

Joséhttp://es.wikipedia.org/wiki/Motivaci\%C3\%B3n

-Test de psicología deportiva:

Conroy, D. E., Elliot, A. J., y Hofer, S. M. (2003). A 2 x 2 Achievement Goals

Questionnaire for Sport: Evidence for factorial invariance, temporal stability, and external validity. Journal of Sport and Exercise Psychology, 25, 456-476.

Recuperado en:http://es.testsworld.net/tag/test-para-

deportistashttp://www.efdeportes.com/efd174/motivacion-en-las-clases-de-

educacionfisica.htm 